\title{
Parentalité et Individualité
}

\author{
Maël LEMOINE
}

Université François Rabelais, Tours

\section{RÉSUMÉ}

Face à l'ensemble des revendications liées aux nouveaux usages de la sexualité et de la reproduction, la notion de parentalité semble appeler une redéfinition. Du point de vue exclusif d'une logique de la liberté, est « parent » l'individu qui a choisi de se définir ainsi en appelant à l'existence un nouvel individu qui bénéficie ipso facto de la même liberté, limitant la sienne. De cette redéfinition découle un droit illimité à la parentalité pour tout individu qui le décide, un droit illimité à l'avortement de toute grossesse non voulue, et une interdiction absolue de I'IVG pour raisons de malformation.

Mots-clefs : parentalité, IVG, procréation assistée, homoparentalité, diagnostic prénatal

De tout ce qu'il peut être donné à un individu d'être, " parent ", c'est-à-dire " père ", mais aussi " frère ", " fils » ou « cousin », est le seul qualificatif qui lui revienne seulement parce qu'un autre individu existe ou a existé, et qui ne puisse lui être retiré, quoi que l'un ou l'autre de ces deux individus fasse ou ait fait. II faut des concitoyens pour être un citoyen, un maître pour être un esclave, une femme pour être un mari, mais certaines actions peuvent défaire ce qui a été fait. Pourtant, rien ne peut défaire ce que le criminel a fait à sa victime - justement, le criminel n'est pas criminel simplement parce que la victime existe, a existé, ou existe encore ; il l'est de par ce qu'il lui a fait. Le Berbère ou le "Caucasien " ne peut pas ne pas l'être, et il ne l'est pas par ce que ses parents ont fait, mais parce que ses parents le sont ou le furent aussi. Assurément, mais il n'est pas ce qu'il est parce que ses parents existent ou existèrent. Par conséquent, de quelque manière que ce soit, tout ce qu'un individu n'est pas par luimême, mais par un autre individu, et tout ce qu'il est de manière irrémédiable du seul fait de l'existence de cet individu, toute cette classe d'attributs qui peuvent lui revenir ne comprend qu'un seul élément : la parenté.

La parentalité est une des formes de la parenté, celle qui caractérise "le père » ou " la mère " par le simple fait que " l'enfant " existe. Bien des difficultés attendent celui qui chercherait à définir la spécificité de la parentalité par la notion d'" engendrement ", comme on le fait habituellement. Aristote, par exemple, écrit que le père et le fils se définissent relativement l'un à l'autre, parce que l'un a « produit » et l'autre a été « produit » [1]. Dira-t-on que les parents d'adoption, les beaux-parents d'un remariage, le couple homoparental, la mère qui a eu recours à une mère porteuse, le père qui a dû se voir substituer un donneur de sperme, etc., sont, d'une certaine manière, les " géniteurs » de l'enfant ? Ce serait en étendre la portée à une signification si symbolique que la notion de génération se viderait de toute sa substance. Une autre possibilité s'offre : définir la parentalité par l'autorité parentale. Aristote le faisait aussi : la paternité est une " autorité royale ", fondée sur l'identité de souche, l'âge et l'affection. Cette deuxième définition est en effet plus large que la précédente, puisque Zeus, dit-il en citant Homère, peut en ce sens être nommé " père des Dieux et des hommes " [2]. Mais dira-t-on que ceux qui exercent légalement, sinon seulement de fait, l'autorité parentale, sont les parents de l'enfant? Ce serait faire abstraction de ce qu'il est des parents qui négligent

\section{Correspondance :}

Maël LEMOINE - U.F.R. Médecine, Département de Sciences Humaines et Sociales, 2 bis Boulevard Tonnelé, 37032 TOURS Cedex 1 - Tel 02.47.36.70.18 -

Fax 02.47.36.61.34 - Email mael_lemoine@hotmail.com 
ou abusent de leur autorité parentale ; or on aura beau lui donner les meilleurs parents adoptifs du monde, il n'en demeurera pas moins que ses parents, si défaillants soientils, et si énergique soit la volonté de l'enfant de les renier, restent bien ses parents.

Ces quelques remarques préliminaires montrent une chose. Le rôle de la réflexion philosophique n'est pas de fixer rigoureusement et définitivement le sens d'un concept. En effet, les usages du langage évoluent de manière imprévisible. Plus une civilisation travaille un mot, plus il devient difficile d'en comprendre le sens précis. La philosophie s'expose donc à une alternative ridicule : chercher à réunir des usages incompatibles sous une définition trop large, ou exclure arbitrairement l'un d'entre eux. Relativiste ou fondamentaliste, elle se prive d'utilité ou de recul. Ici, la notion d'engendrement a incontestablement défini d'abord la parentalité. Mais la révolution génésique amorcée au XXe siècle dans le champ scientifique d'une part, avec les pratiques de contraception et d'avortement, de fécondation artificielle et de clonage, dans le champ culturel de l'autre, avec de nouvelles approches de la sexualité, de l'union parentale, a sans cesse déplacé les frontières d'une conception simple et immédiate de l'engendrement. En cherchant à dériver tous les modes de la parentalité de son mode le plus simple, on se dissimule les changements profonds qui affectent en retour la conception de la parentalité.

On se demande ici s'il ne vaudrait mieux pas tenter une autre approche. Parmi toutes les formes de parenté, la parentalité se signale par une caractéristique remarquable, quelles qu'en soient les formes : c'est la seule relation de parenté qui, désormais, se choisit. Personne ne choisit d'être un fils, un frère, un cousin, mais personne ne peut plus se voir imposer d'être parent. On reconnaît aujourd'hui le droit à la femme violée de renoncer à l'enfant qui pourrait naître. Puisque le choix est reconnu, celle qui estime ne pas avoir ce droit choisit encore. Or, si le choix est une caractéristique nouvelle de la parentalité, c'en est incontestablement la spécificité. Être " père " ou " mère ", c'est donc avoir choisi d'être désormais défini comme tel par l'existence d'un "enfant", sans jamais plus pouvoir revenir en arrière, même s'il ne suffit pas d'avoir choisi. La nature n'impose plus d'avoir des enfants et, de moins en moins, impose-t-elle encore de n'en pas avoir. C'est d'elle, et contre l'usage des nations, que se réclamait Edmond le bâtard dans la tragédie du Roi Lear, Edmond que son père a pris tant de plaisir à concevoir, bien qu'il soit venu avant qu'on ne l'appelle [6]. En prenant le contrôle de l'engendrement, l'homme déplace les critères de la parentalité, qui n'est plus affaire de nature, mais affaire de liberté.

On touche là à un point essentiel. II est rare que les prises de position reposent sur une seule ligne de raisonnement, conduite rigoureusement depuis son principe, et développées dans toutes ses conséquences. Les lois, les consensus mêlent souvent des logiques distinctes, voire opposées, et même incohérentes. Ainsi en va-t-il des conceptions "naturalistes " et " libérales " de la parentalité, les unes anciennes, les autres modernes. Désormais que la nature est dépouillée de toute présomption d'intention, elle est conçue comme un générateur aléatoire. Au contraire, la liberté est le règne de l'intentionnalité. Ce qui est naturel n'a pas été voulu, ce qui est libre l'a été. A la vérité, la parentalité n'a jamais été la seule affaire de la nature, et il s'y est toujours mêlé une part de liberté. Le peuple inventeur de la démocratie, qui jalousait tant sa liberté, exposait aussi les enfants difformes ou non désirés, et pratiqua la sodomie, dit-on, pour éviter la dilapidation des héritages. La science retire progressivement à la nature le privilège de fixer des limites à la liberté d'engendrer, mais elle n'a jamais retiré à la nature le privilège de fixer des limites à la liberté de ne pas engendrer. L'engendrement n'a jamais été pas rigoureusement l'affaire de la nature, car jamais elle n'imposa l'enfant, si l'homme ne lui reconnaissait lui-même le droit de l'imposer. Peut-être le principe de la liberté qui semble prévaloir aujourd'hui dans la définition de la parentalité, n'est-il pas suivi jusqu'au bout, et se mêle-t-il aussi de naturalisme ? Peut-être s'agit-il d'une simple incohérence accidentelle, peut-être, au contraire, d'une profonde nécessité, comme si liberté des individus et parentalité s'excluaient ? Pour répondre, il serait éclairant de chercher à développer pour elle-même la ligne des conséquences qui découlent du principe de liberté, dans le domaine de la parentalité.

La liberté moderne se définit par deux exigences : elle est universelle, et elle est absolue. C'est-à-dire, d'une part qu'elle appartient à toute entité capable de l'exercer, et, de l'autre, que rien ne doit la limiter qu'elle-même. Celui qui exerce, peut exercer ou exercera la liberté est appelé individu. II peut tout faire, sauf limiter la liberté d'un autre individu : "sa liberté, dit-on, s'arrête où commence celle des autres. » Cette formule est l'indice d'un problème plutôt que d'une solution : où celle des autres commence-t-elle donc? C'est là qu'intervient la nécessité de légiférer. Kant écrit : " le droit est la limitation de la liberté de chacun à la condition de son accord à la liberté de tous, en tant que celle-ci est possible selon une loi universelle ; et le droit public est l'ensemble des lois extérieures qui rendent possible un tel accord universel. » [4]. La loi, qui limite la liberté, a désormais besoin d'être justifiée, à cause de la reconnaissance du principe universel de liberté. Tant que ce n'était pas le cas, elle pouvait se contenter de frapper d'interdit et de rendre impossible un champ d'action sans que cela ne profite à la liberté de quiconque - d'autres objectifs fondaient la loi, comme éduquer l'homme, plaire à Dieu, respecter les « lois de la nature ". Aujourd'hui, la législation moderne ne se justifie que dans la mesure où elle protège la liberté des individus : elle ne peut limiter la liberté des uns que pour protéger celle des autres. Cela laisse place à un nombre indéfini de législations " libérales " possibles - d'où les conflits. Cependant, il est un point invariable dans toutes ces législations possibles. Tout individu voit sa liberté limitée par le respect qu'il doit à l'existence d'un autre individu.

Or la parentalité, qui est en quelque sorte l'appel à l'existence d'un individu par un autre individu, consiste donc à vouloir la limitation de sa propre liberté. On choisit librement d'être parent tant qu'on ne l'est pas encore, mais une fois devenu parent, l'existence de l'enfant s'impose. Le règne de la liberté ne signifie qu'on s'est libéré des contraintes de 
la nature, que pour supporter les contraintes de la liberté. Si la libéralisation de l'engendrement rencontre des limites, c'est donc dans la liberté elle-même plutôt que dans la nature. En effet, l'individu engendré dispose en droit de la même liberté que l'individu qui l'engendre. Le Père n'a plus, comme dans la Rome de la République, droit de vie et de mort sur ses enfants. Pas plus ne peut-on reconnaître sans contradiction aux parents, comme à aucun autre individu, le pouvoir de décider ce qu'il faut, ou non, tenir pour individu. Car, face à un autrui qui lui ferait obstacle, celui qui voudrait s'imposer absolument n'aurait plus qu'à lui nier toute individualité, et la liberté se détruirait d'elle-même. C'est le premier problème rencontré par le développement du principe libéral. Si l'homme ne peut donc pas décider de l'individualité, ne lui reste-t-il pas qu'à laisser cette prérogative à la nature ? De là découle la question problématique : à quel moment l'engendrement a-t-il produit un nouvel individu ? Quand l'individualité commence-t-elle ? Sitôt que l'engendré est reconnu individu en effet, les parents, devenus parents, deviendraient criminels en l'éliminant. Ainsi commence le règne de la loi, c'est-à-dire le règne de la liberté. Auparavant s'étendait le règne de la nature, dans l'intervalle entre l'acte naturel d'engendrement et la reconnaissance de l'individualité du vivant engendré. C'est dans cet intervalle qu'on reconnaît aux parents une liberté sans frein, puisque c'est contre la nature qu'elle s'exercera, et non contre la liberté elle-même.

Mais laisser à la nature le privilège de décider de l'individualité des individus, c'est encore se poser un problème insoluble. Voici le raisonnement que l'on suit généralement. L'individu est celui qui est capable d'exercer sa liberté - et qui en est capable, en a le droit. En être capable, c'est choisir, et choisir, c'est penser. Mais quand la pensée commence-t-elle ? Au plus tôt, dès que les cellules nerveuses la rendent possible. Puisque nous serions choqués des conséquences de l'opinion que c'est seulement à l'âge de 6 ans, de 2 ans, de 6 mois, de 3 mois, et même à 6 mois de gestation, voire que certains, ne pensant jamais, ne seraient pas des individus, nous cherchons d'autres critères, et interprétons d'une autre manière, à savoir en termes neurophysiologiques, cette liberté " potentielle ", cette pensée " en puissance ". Mais que ferons-nous du nouveau-né anencéphale ? Est-ce un individu ? D'un point de vue strict, il ne l'est pas ; mais qu'opposerait-on aux parents qui s'y seraient attachés comme à un fils ? Faut-il leur refuser le fait qu'ils sont parents? Peut-on leur assurer que jamais la science, qui pour l'instant maintient seulement en vie l'être auquel ils ont donné naissance, ne pourra lui donner le pouvoir de penser avant qu'il ne devienne inéluctable qu'il meure ? La difficulté, ici comme dans d'autres problèmes rencontrés par la médecine, tient à ce que la science, cherchant à trouver des critères de reconnaissance objectifs pour délimiter des faits que seules des valeurs ont définis, s'expose à la même alternative que celle décrite plus haut à propos de la philosophie. Dans un autre domaine, par exemple, la science croit tenir une définition rigoureuse de la mort, qui balaie les conceptions vagues du sens commun. Mais ce n'est qu'un critère, que la définition prétendument vague balaie sitôt qu'il est remis en cause par l'expé- rience d'un cas exceptionnel. En effet, du temps où l'on définissait la mort par arrêt cardiaque, il fallait bien reconnaître que la définition du sens commun s'imposait devant le cas d'un homme dont le coeur s'était arrêté puis était reparti. II peut toujours en être de même pour tout critère retenu aujourd'hui. Bichat avait déjà précisé le processus et les formes de la mort en distinguant l'arrêt respiratoire, l'arrêt du coeur et l'arrêt du cerveau [3] : ce n'était pas redéfinir la mort elle-même.

II faut donc proposer ici d'examiner à nouveau la notion d'individualité. On a dit qu'avant que l'individu ne soit, même s'il y a peut-être déjà eu génération, le choix d'être parents ne concerne que les parents. C'est donc le choix de s'engager, ou de ne pas s'engager, dans la parentalité, de devenir parents si rien ne l'empêche, ou de ne pas le devenir. Mais si celui qui est parent a nécessairement choisi de l'être, une fois qu'il l'est devenu, il ne peut plus ne pas l'être ou, du moins, ne pas l'avoir été. La parentalité est un choix qui, pour appartenir absolument à l'individu, présente des conséquences qui lui échappent absolument, car il y va de l'existence d'un nouvel individu. Puisque devenir parent est un choix, ne faut-il pas, dès lors que ce choix a été fait, tenir l'existence de l'enfant pour légitime, qu'il ait, ou non, une conscience, une volonté, etc. ? D'un côté, ne faudraitil pas accepter ce paradoxe que les parents décident absolument de l'existence d'un nouvel individu ? De l'autre, ne faudrait-il pas accepter qu'une fois engagé, ce choix soit irréversible, quoi qu'il advienne ? Décider de l'existence d'un nouvel individu qui n'existe pas encore, ce n'est pas décider de l'individualité d'un individu qui existe déjà ; ce n'est pas même décider qu'il existera, c'est décider de s'engager dans la voie qui conduira, peut-être, à son existence. Sitôt que cette volonté des parents est incarnée dans un vivant, ce vivant doit dès lors être appelé individu : car l'existence d'un individu commence dans la rencontre d'une intention des parents avec une réalisation effective. L'embryon qui porte l'intention des parents est déjà un individu, car une liberté lui est déjà reconnue par les libertés qui l'ont engendré. C'est ce qui conduit la mère ayant perdu un foetus de 5 mois dans un accident de voiture à demander que le chef d'inculpation " homicide " soit retenu, alors qu'elle aurait pu demander même à cinq mois la fin d'une grossesse non désirée. II ne s'agit pas d'incohérence, comme si, d'un côté, on reconnaissait la logique de la vie, de l'autre, une logique de mort : c'est bien la cohérence de la liberté et de l'individualité.

Cependant, pour suivre la logique de l'individualité jusqu'au bout, il est impossible de reconnaître aux parents le droit de refuser d'incarner leur intention de procréer dans ce corps$\mathrm{ci}$, précisément, qui se forme, quelle qu'en soit la raison une malformation, par exemple. En effet, si c'était le cas, on ouvrirait la possibilité d'une individualité perpétuellement sursitaire - celle d'un enfant né, qui grandit, vit sans que ses parents ne l'aient encore reconnu comme un individu, et donc sans que personne ne soit fondé à le leur imposer comme tel. Il serait alors une simple propriété. Or cette possibilité ne pourrait être limitée que par un recours à un principe " naturel » : la sympathie, par exemple, celle même qui 
nous fit finalement renoncer à l'esclavage, celle qu'il faut étouffer pour y recourir. En effet, l'individualité commence dans une rencontre de l'intention parentale et de la matière qui l'incarne, quelle que soit cette matière - il suffit pour cela que son développement conduise à l'existence d'un individu, c'est-à-dire d'une capacité à exercer la liberté, laquelle ne repose pas seulement sur les choix opérés en conscience, mais s'exprime déjà dans le développement biologique lui-même. Vivre est une liberté. Les parents ont le pouvoir d'appeler un individu à l'existence et celui de refuser à un être le droit d'être individu, à condition que cela concerne n'importe quel être possible. Mais ils n'ont pas le droit de choisir quel être sera reconnu comme un individu, car tous ceux qu'ils refuseraient, ils les auraient préalablement voulus, c'est-à-dire conçus comme des individus. Ces conséquences s'imposent du moins dans une logique pure de la liberté - et l'on voit comment les pratiques reconnues aujourd'hui n'en découlent pas exclusivement.

Du point de vue de l'enfant, deux remarques s'imposent. D'abord, on ne saurait dire que l'enfant a été mis au monde sans son consentement, comme l'affirme Kant [5]. Si l'enfant n'est pas un individu avant d'exister, il n'y a pas de volonté pour consentir ; quel sens cela a-t-il d'affirmer qu'il aurait pu ne pas vouloir naître ? De là, il ne découle pas que l'existence de tout individu, si libre qu'il puisse devenir, comporte une irréductible part de nature (natura), en ce qu'il est né (natus). Car ce n'est pas la nature qui l'appelle à l'existence, ce sont des individus. Ce n'est donc pas le défaut de consentement de l'enfant qui donne un devoir à ses parents, c'est son existence lui-même - c'est le fait de l'avoir voulue. En revanche, l'enfant peut vouloir ne pas être né - ce qui n'est pas : avoir voulu ne pas naître. II n'a aucun droit à reprocher à ses parents sa propre existence, car il n'était pas alors une personne. De là découle la seconde remarque : on ne saurait affirmer que le fait de provenir de la volonté d'individus, et non de la nature, est une limitation de la liberté de l'enfant. Ses parents n'ayant, dans une logique de la liberté, aucun droit à définir ce qu'il est, mais seulement à décider qu'il pourra être, ils lui rendent possible l'exercice même de la liberté. Quand bien même, dans une logique mixte, ils auraient un droit, limité ou intégral, à choisir ce que sera l'individu qui vivra, ils ne feraient en cela que se substituer à la nature pour décider de ses caractères innés. Or, que la nature ou que des parents les aient déterminés, ils s'imposent à lui tout autant, et, même dans le second cas, cela ne le fonde pas à reprocher à ses parents ce qu'il est - puisque cela relevait exclusivement de leur décision avant qu'il ne soit un individu.

Enfin, il découle de ce principe intentionnel de la parentalité qu'aucun foetus, ni même aucun enfant dont l'existence n'a été voulue à aucun moment ne pourrait se voir reconnaître la légitimité d'individu. Une femme qu'on aurait violée, puis forcée à accoucher pourrait ainsi, semble-t-il, refuser l'existence d'un enfant qui désormais, existe. Mais en réalité, celui qui l'aurait forcée à le garder serait réputé en être père (ou mère) : car il ne serait pas requis que deux individus aient voulu son existence, un seul y suffisant. Et cette volonté de l'existence d'un individu est réputé être amour.
Voilà donc à quelle situation conduit le principe de liberté souveraine, c'est-à-dire le principe de l'individualité. II semble bien qu'il s'impose progressivement dans toutes les dimensions de l'existence, et n'aura de cesse qu'il ne les ait toutes investies, ou qu'il rencontre un insurmontable obstacle. Le but de cette réflexion n'était ni d'être consensuelle, ni de s'engager, mais de décrire une hypothèse. S'il s'était agi de s'engager, elle mécontenterait les partisans de la " liberté totale de procréer ", incluant le choix de mettre fin au développement d'un embryon pathologique, comme si la grossesse elle-même était anormale ou pathologique. Mais elle mécontenterait aussi les partisans de "la vie ", puisque l'engagement en faveur d'une liberté universelle souveraine ouvre un droit illimité à l'avortement de grossesse non voulue. S'il s'était agi enfin de brosser un tableau complet de la parentalité, il eût encore fallu en peindre la joie et les angoisses, montrer comment l'une est liée à l'émerveillement devant la beauté de cette totalité des possibles qu'il représente, comment l'autre est liée à la limitation de ces possibles et à l'anéantissement totale de son existence ; il aurait fallu encore décrire l'amour et la peur de l'enfant, comment le premier s'attache à son absolue singularité davantage qu'à sa ressemblance, que la seconde repose sur son étrangèreté qui s'impose pourtant aux parents comme devant être aimée. Car rien ne peut pousser une liberté à se limiter par une autre liberté, si ce n'est l'amour.

\section{REFERENCES}

\section{ARISTOTE : Métaphysique. ?, 15, 1021a : 23-25.}

2. ARISTOTE : Politiques. I, 12, 1259b : 10-17.

3. BICHAT $X$. : Recherches physiologiques sur la vie et la mort (1801). Paris, Bechet Jeune, 1822.

4. KANT E. : Sur l'expression courante : il se peut que ce soit juste en théorie, mais en pratique, cela ne vaut rien (1793). Paris, Vrin, trad. L. Guillermit, $1992: 30$.

5. KANT E. : Métaphysique des moeurs. Première partie. Doctrine du droit (1796), § 28.

6. SHAKESPEARE W. : The Tragedy of King Lear. I, 2, 1-6 ; I, 1518.

Communication au XIII Séminaire de la Fédération Française des CECOS,

Tours, 7-9 Avril 2005.

Manuscrit reçu : mars 2005 ; accepté mai 2005. 
Parenthood and Individuality

Maël LEMOINE

The concept of parenthood needs to be redefined in relation to the new uses of sexuality and reproduction. Exclusively from the view of point of personal freedom, a "parent" is any person who has chosen to define himself/herself in this way with reference to the existence of another individual, who ipso facto benefits from this same freedom, limiting his/her own freedom. This redefinition is derived from an unlimited right to parenthood for any individual who chooses parenthood, an unlimited right to abortion for any unwanted pregnancy, and absolute prohibition of abortion for congenital malformations.

Key words: parenthood, abortion, IVF, same-sex parenthood, selective abortion, pre-implantatory diagnosis 